\title{
Frank Bardelle \\ Jenseits des Atlantiks - Zur Kritik der eurozentrischen Kultur- und Kolonialgeschichtsschreibung
}

\begin{abstract}
»Die Wahrnehmung, die der abendländische Mensch von seiner Zeit und seinem Raum hat, läßt eine Struktur der Ablehnung erscheinen, von der aus man eine Rede denunziert, indem man sagt, sie sei nicht Sprache, eine Geste denunziert, indem man sagt, sie sei nicht Tat und eine Gestalt denunziert, indem man sagt, sie habe kein Recht, in der Geschichte Platz zu nehmen.«
\end{abstract}

Michel Foucault

Zusammenfassung:Das >Euro-Fieber, das spätestens im Jahre 1992 seine höchsten Temperaturgrade erreichen wird, scheint Ausdruck einer Art Infektionskrankheit zu sein, die im 15./16. Jahrhundert ihren Ausgang nimmt und zu eigenartigen Restriktionen der Wahrnehmung führt, die der europäische Mensch in bezug auf den Rest der Welt und auf seine eigene Tradition und Geschichte entwickelt. So wird auch die Kolonisation der transatlantischen Räume von Anfang an und bis auf den Tag nahezu ausschließlich nach den Maßgaben einer imperial und merkantil inspirierten Rationalität gewichtet und bewertet. Der Artikel setzt sich zum Ziel, die gängigen Perspektiven der eurozentrischen historiographischen Konstruktionen und Rekonstruktionen zur ,Entdeckung ' und 'Eroberung, Amerikas ideologiekritisch zu durchleuchten und liefert Ansätze für eine erweiterte und adäquatere Sichtweise.

\section{Vorentdeckungen}

Ende November 1982 erschütterte die an offen ausgetragenen Kontroversen nicht arme UNO-Vollversammlung ein heftiges Wortgefecht. Sechsunddreißig Nationen, unter ihnen die Vereinigten Staaten in seltener Eintracht an der Seite von Kuba und Nicaragua, hatten gemeinsam den Antrag eingebracht, die Vereinten Nationen mögen für den 12. Oktober 1992 eine würdige Gedenkfeier vorbereiten. An diesem Tag nämlich wird sich die Landung des Christoph Columbus auf der Karibikinsel Guanahani zum 500. Male jähren, ein Ereignis, das offiziell und gemeinhin als die Entdeckung Amerikas gilt. Das Vorhaben der Planung und Durchfülırung eines solchen Jubiläumsaktes stie $ß$ bei den Delegationen Irlands und Islands auf entschiedenen Widerspruch. In zum Teil höchst polemischen Debattenbeiträgen wetterten Vertreter der beiden Länder gegen die maßlose Arroganz und schnodderige Selbstverständlichkeit, mit der die spanischsprachige Welt und die amerikanischen Staaten die Entdekkung Amerikas für Columbus beanspruchen. Die Iren führten den Heiligen Brendan ins Feld. Der fromme Mönch soll zusammen mit 17 Gefährten auf der Suche nach dem 
verlorenene Paradies in einem kleinen Fischerboot von Irland aus westwärts gesegelt sein und nach siebenjähriger Irrfahrt seinen Fuß bereits 700 Jahre vor dem Genuesen in die Neue Welt gesetzt haben (vgl. O'Donaghue 1893). Die Isländer verwiesen auf die Expeditionen Eriks des Roten und Leif Erikssons zum nordamerikanischen Festland, Unternehmungen, die um das Jahr 1000 nach Christus datieren und zu lockeren Verbindungen zwischen beiden Hemisphären führten, die vermutlich bis Mitte des 14. Jahrhunderts andauerten (vgl. Jones 1965). Der Streit in der Vollversammlung nahm derartig hitzige Formen an, daß sich Versammlungspräsident Imre Hollai aus Ungarn genötigt sah, den Tagesordnungspunkt vorerst abzusetzen, damit außerhalb des Plenums ein Kompromiß gefunden werden könne.

Skizzierte Auseinandersetzung steht in einer Tradition, die bis zum erfolgreichen Abschluß der ersten Reise des Columbus zurïckreicht. Der wagemutige Seefahrer thronte noch nicht einmal auf dem Sockel des Entdeckers einer $>$ Neuen Welt $<$, da setzten bereits vehemente Versuche ein, ihm diesen Platz streitig zu machen. So wurde die Mär von dem portugiesischen Piloten Alonso Sanchez kolportiert, der angeblich 1483 oder 1484 in Amerika gelandet ist. Auf dem Weg von Spanien nach England durch widrige Winde abgetrieben, segelte er wochenlang nach Westen, bis er auf mehrere Inseln stieß, die er Indien zurechnete. An Land begegneten Alonso und seine Leute nackten Menschen. Die Eingeborenen empfingen die Europäer freundlich und versorgten sie großzügig. Lediglich Alonso und drei oder vier Matrosen fanden schließlich den Weg zurück nach Portugal. Vielen Spaniern galt nicht der Italiener Columbus, sondern ihr Landsmann Martin Pinzón, der 1492 Kapitän der >Pinta< war, als eigentlich herausragende Figur und treibende Kraft des Entdeckungsunternehmens. Pinzón habe die Idee zu der Fahrt gehabt. Von Pinzón seien die maßgeblichen Informationen gekommen, auf die er in den Bibliotheken des Vatikan gestoßen sei (vgl. Henning 1940: 5-19).

Solche und ähnliche eher kleinkarierte Reklamationen und die kontroverse Diskussion ihrer aktuellen Varianten verstellen gelegentlich den Blick für den Umstand, daß es in Europa Kenntnisse um die Existenz von Landmassen jenseits des Atlantiks gibt, die über das Mittelalter bis zum Altertum dokumentiert sind. Schon in Texten der römischen und griechischen Antike (Seneca, Pomponius Mela, Strabo, Erastosthenes, Aristoteles) findet sich die Kugelgestalt der Erde beschrieben und die Ansicht vertreten, daß man mit Ostwind von Sonnenaufgang segelnd in durchaus vertretbarer Zeit auf weitere bewohnte bzw. bewohnbare Erdteile stoßen wird (vgl. auch Henning 1940: 170 ff.) Plutarch vermerkt in seinen >Maralia< u.a., daß >die Barbaren< von einem ausgedehnten Festland in Richtung des sommerlichen Sonnenuntergangs fern im weiten Meer >fabeln< (vgl. Henning 1940: 171). Hier hat sich offenbar die Geographie mündlicher Kulturen in einer schriftlichen Struktur verfangen, die durchaus realistische - wenn auch mitunter mythisch verschlüsselte - Beschreibungen und Angaben zu Unrecht als Lügenmärchen diskreditiert. Darüber hinaus bieten die schriftlichen Niederschläge sicher nur einen schwachen Abglanz dessen, was >die Barbaren< über die Kontinente und Meere der Erde tatsächlich wußten. Die schriftlichen Traditionen um $>$ unbekannte $<$ Erdteile auf der anderen Seite der Welt finden bei mohamedanischen 
und christlichen Autoren des Mittelalters (Masudi, Edrisi, St. Augustinus, Albertus Magnus, Roger Baco) ihre Fortsetzung. Die mündlichen Traditionen verlieren sich in archaischen Nebeln.

Beobachtungen und Befunde der Archäologie, der Ethnologie und der Linguistik lassen heute den Schluß zu, daß wahrscheinlich bereits im Jungpaläolithikum Verbindungen zwischen Nordamerika und Westeuroasien bestanden. Strittig ist die Frage, welcher Art diese Verbindungen waren. Die allgemein anerkannte Theorie behauptet, die Ureinwohner Amerikas seien während der letzten Eiszeit, die vor etwa zweihunderttausend Jahren begann und vor etwa zehntausend Jahren endete, aus Sibirien über die Beringstraße nach Alaska eingewandert. (Durch die in der ausgedehnten Eiskappe gebundenen Wassermassen sei der Meeresspiegel so niedrig gewesen, daß in der Beringstraße eine Landbrücke beide Hemisphären verband.) Über die Gletscher und durch die Tundra Alaskas drangen die eiszeitlichen asiatischen Sammler und Jäger immer weiter in südlicher Richtung vor, bis sie schließlich in Gegenden Fuß faßten, die dann jeweils zu ihren $>$ Stammlanden $<$ wurden. Nach etlichen Jahrtausenden fand sich so in sukzessiven Schüben der gesamte Doppelkontinent bis an die äußerste Spitze Südamerikas bevölkert. Hat die postulierte Landbrücke in der Beringstraße jemals existiert und ist sie tatsächlich beschritten worden, dann liegt die Annahme von $>$ Gegenverkehr $<$ nicht fern, dann müssen auch Menschen und Informationen von Amerika zuriick nach Asien und von dort weiter in europäische Regionen gelangt sein.

Nun hat keine der relevanten Ausgrabungen in Alaska und in Nordostasien bisher einen einzigen überzeugenden Beweis für Wanderungen über die Beringstraße von Sibirien aus ans Licht gefördert (vgl. Müller 1982). Dem entspricht, daß auch keine der überlieferten indianischen Stammesgeschichten Anhaltspunkte bietet, die als Erinnerung an einen solchen Marsch gedeutet werden könnten (vgl. Steiner 1985: 143f.). Weit eher verweisen die auffindbaren Spuren auf einen gemeinsamen Ursprung der Altkulturen Amerikas und Eurasiens in Zentren, die um den borealen Rand des nördlichen Eismeeres zu suchen sind. Von hier aus liefen dann Wanderungswellen sowohl nach Süden (Amerika) wie nach Südwesten (Eurasien). In diesem Zusammenhang sind u.a. die sprachlichen Verbindungen zwischen dem Eskimoidiom und dem Indogermanischen erwähnenswert, die selbst Skeptiker anerkennen (vgl. Müller 1982). Weiter gilt es in der Paläoanthropologie als unumstritten, daß die ersten Sapientes, die ohne > Vorstufen $<$ um 42.000 vor Christus plötzlich in Europa auftauchten (und möglicherweise den Neandertaler in derartige Minderwertigkeitskomplexe trieben, daß er ausstarb), durch nördliche Ausstattung und Lebensweise gekennzeichet waren. Ferner sind boreale Herkunftsmythen beiderseits des Atlantiks manifest (vgl. Müller 1982).

Angesichts der skizzierten Befunde erscheint es verwunderlich, daß bis heute so hartnäckig an der Beringstraßen-Theorie festgehalten wird. Dieser Umstand kann als Ausdruck einer eurozentrischen Voreingenommenheit der gesamten Kulturgeschichtsschreibung gelten, die eine Evolution der menschlichen Spezies postuliert, die in der Alten Welt ihren Uisprung nimmt. Die nachweisbaren Altmenscharten vom 
primitiven Pithecanthropus über den Neandertaler bis zum modernen Sapiens hat man in einer fortschreitenden Kausalkette miteinander verbunden und dementsprechend den Schluß gezogen, daß die Frühgeschichte des Menschengeschlechts nur dort anzusiedeln ist, wo Überreste der Altmenschentypen festgestellt werden konnten. Da Amerika solche nicht vorzuweisen hat, muß es mithin von Eurasien aus bevölkert, ja regelrecht befruchtet worden sein. Im Licht einer Reihe jüngerer Funde läßt sich allerdings die genetische Ableitung der Sapientes von den Altmenschen kaum weiter aufrecht erhalten.

Weit angemessener sind die altsteinzeitlichen Menschenarten nicht hintereinander, sondern - mit entsprechenden Phasenverschiebungen - chronologisch nebeneinander zu verorten. Das Alter des Sapiens nämlich muß weit höher veranschlagt werden, als ursprünglich angenommen. Seine Entstehungsgeschichte, die lange in der Zeit vor 100000 Jahren vermutet wurde, sucht man heute eher in der Zeit vor 600000 Jahren. Nách Anpassung der Phylogenese an die neuesten Erträge archäologischer Ausgrabungen besteht kein zwingender theoretischer Grund mehr, kategorisch auszuschlieBen, daß die Frühgeschichte der Spezies Mensch auch in Amerika stattgefunden haben könnte, da Spuren von Altmenschentypen als notwendige Voraussetzung für eine solche Annahme entfallen. Mehr noch: Es liegen durchaus Anhaltspunkte vor, die es gestatten, Amerika als ältesten Sapienskontinent in Betracht zu ziehen. So wurde im Jahre 1966 in Californien in einer Kiesschicht unter vulkanischen Tuffen, die von tertiären Ausbrüichen der Sierra Nevada herrühren, ein menschlicher Schädel entdeckt. Die Fundumstände, die diesen ehrwürdigen Knochen als ältesten bislang aufgetauchten menschlichen Überrest überhaupt erscheinen lassen, erfuhren seinerzeit mehrfache Überprüfung, die zu Zweifeln keinen Anlaß gab. Bei einer anderen Ausgrabung stießen Wissenschaftler des U. S. Geological Survey in einem alten mexikanischen Flußbett auf einige relativ hoch entwickelte Werkzeuge, deren Alter - nach den gängigeı Methoden bestimmt - bei 250000 Jahren liegt (vgl. Steiner 1985: 143). Lediglich das Diktat der eurozentrisch geprägten Evolutionstheorie führt dazu, solche Resultate ernsthafter archäologischer Arbeit und Analyse für unmöglich zu erklären, als Schwindel zu dikreditieren oder schlicht kommentarlos zu ignorieren. Der Glaube an den Mythos eines > fremden < Ursprungs des amerikanischen Menschen, der sich bis heute hartnäckig hält und vor allem im Feld der >Schulgeschichte und unbekümmert propagiert wird, reicht fünf Jahrhunderte zurück. Für Nachfahren aller möglichen Völker und Kulturen wurden die amerikanischen Ureinwohner seit dem 16. Jahrhundert ausgegeben, für Juden, Karthager, Phönizier, Ägypter, Babylonier, Kelten, Afrikaner, Waliser, Chinesen, Japaner und für Inder gar: Nur aus Amerika selbst konnten und durfte1 sie nicht stammen. Das widersprach jeglicher vom Bewußtsein abendländischer Überlegenheit durchtränkten `Entdeckermentalität $<$. Für Amerika mußte eine seuropäische< Schöpfungslegende her, die in der Folge manch wunderliche Blüten trieb bis hin zur These grundsätzlicher Inferiorität der gesamten amerikanischen Flora und Fauna, die letztlich nur mehr oder weniger degenerierte und verunglückte Kopien der europäischen zu bieten habe (vgl. Rehrmann in PROKLA 75). Erst durch die segensreichen Auswirkungen europäischer Ideen und 
europäischer Technik sei Amerika überhaupt in menschenwürdiger Weise bewohnbar geworden. In dieser Tradition ist die Beringstraßen-Theorie zu sehen, in dieser Tradition steht bis auf den heutigen Tag die auf Amerika bezogene europäische Geschichtsschreibung, wie noch eingehender zu zeigen sein wird.

Unabhängig davon aber, welche orthodoxen oder unorthodoxen Erklärungsansätze man immer auch favorisieren mag, die Fülle amphiatlantischer Gemeinsamkeiten in den Gerätesätzen, den >Architekturen<, den Sprachen, den Mythologien liegt auf der Hand und steht offensichtlich jenseits aller Zufälle. »Die beiden Kontinente passen bruchlos zusammen « (Müller 1982: 109). Vor skizziertem Hintergrund erscheint die Vermutung keineswegs abwegig, daß vor der >Tat des Columbus< diesseits wie jenseits des Atlantiks ein Wissen um die jeweilige andere Seite vorhanden war, das bis an den Grund der Zeiten reicht, sich über beide Hemisphären gleichermaßen ausbreitete und trotz vielfältiger Wandlungen, Barrieren, Kolonisierungen, Um- und Neuprägungen und Enteignungen durch die Jahrtausende nie gänzlich aus dem Gedächtnis der Menschen verschwand. Verdichtet zu Sagen und Legenden, in mehr als rudimentären Partikeln >inkorporierter < Teil des >kollektiven Unbewußten<, hat dieses Wissen zweifellos Einfluß auf die Wünsche, Hoffnungen und Sehnsüchte vieler derjenigen, die ab 1492 ihren Weg nach Amerika suchen. Den Geschichten, die sich in der Alten Welt vor 1492 (und allem Überlegenheitswahn und allen Inferioritätsphantasmen zum Trotz auch noch lange danach) um die Neue Welt ranken, ist vor allem eines gemeinsam: Sie vermitteln und nähren die Vorstellung von einem Land, in dem eine üppige Natur ihre Gaben im Überfluß spendet und sich Menschen ohne Fron und knechtisches Mühen - und ohne Staat zu machen - in autonomer Kompetenz selber versorgen können.

\section{Spekulanten und Investoren}

Im Jahre 1607 verfaßt ein gewisser George Percy einen Bericht über seine $>$ Reise nach Virginia< und über die $>$ ersten Tage $<$ der dortigen englischen Kolonie (vgl. Billings 1976: 22-26). Enthusiastisch schildert der Autor die beeindruckende Fülle an pflanzlicher und tierischer Nahrung, die das Land naturwüichsig zu bieten hat. Von Austern ist die Rede, die groß und köstlich sind, vom überquellenden Fischreichtum der Flüsse, von allen möglichen Beerenarten, bekannten und unbekannten, >viermal besser< als das, was England an Vergleichbarem aufweisen kann, von Wein, der in schweren Trauben an vielen Bäumen wächst, von Nüssen, Kirschen, Truthähnen und Vogeleiern, von guten und fruchtbaren Böden. Doch wird auch notiert, daß die Kolonisten knapp an Lebensmitteln waren und erschreckend viele Personen im Verlauf der ersten zwölf Monate vor allem an Hunger zugrunde gingen. Die Frage liegt auf der Hand, wie dieser - zumindest für die Engländer betrübliche - Umstand mit dem beschriebenen einheimischen Nahrungsdargebot in Einklang zu bringen ist. Percys Bericht läßt vermuten, daß sich die Kolonisten in ihrer Mehrzahl an einem offenbar schlecht gewählten Ort regelrecht einigelten und sich so zu künstlicher Bewegungslosigkeit 
verdammten, ein Verhalten, das die Nutzung der ökologischen Potentiale und Ressourcen des Raumes für eine effektive Selbstversorgung hinderte. Gelegentliche Querelen und Scharmützel mit den Indianern, in die bestimmte Kolonisten wiederholt und kaum ohne eigenes Verschulden gerieten, mögen diese >Bunkermentalität gefördert haben.

Die von Percy akribisch geführte chronologische Sterbeliste, die zum überwiegenden Teil auch die Berufs- bzw. Standestitel der Verblichenen verzeichnet, vermittelt den Eindruck, daß der Tod vorrangig die nach europäischen Maßstäben bessergestellten >Herrschaften< ereilte. Ein anderes Dokument aus dem Jahre 1610 (vgl. Billings 1976: 28 f.) berichtet, daß vor allem die »commanders « und »officer's von aus Europa mitgebrachten Vorräten lebten und dabei in relativ kurzer Zeit bis zur völligen Ausrottung u.a. Hühner, Ziegen und Schafe verspeisten, Haustiere, die unter einer längerfristigen Perspektive im Stall und auf der Weide für das leibliche Wohl aller Kolonisten weit nützlicher hätten sein können als in den Kochtöpfen und an den Bratspießen der Elite. Das >einfacher Kolonistenvolk dagegen, so ịst zu lesen, ernährte sich hauptsächlich von (in jenen Jahren noch schadstoffreien) Wurzeln, Kräutern, Eicheln, Bucheckern, Walnüssen, Beeren und Fischen, eine in ernährungsphysiologischer Hinsicht mehr als hinreichende und keinesfalls ungesunde Komposition, deren Bestandteile - in Verbindung mit fachkundiger Lagerung und Konservierung - nicht so ohne weiteres ausgehen konnten und jederzeit frei verfügbar waren. (Man wird es als Ausdruck spezifischer und typischer Vermessenheit europäischen Zuschnitts werten müssen, wenn solch eine $>$ Speisekarte $<$ - damals wie heute - als mehr oder weniger erbärmlich diskreditiert wird.) Einige Kolonisten gingen, wie Percy erzählt, in der Sorge für sich selbst einen entscheidenden Schritt weiter. Sie >verschwanden< einfach, d.h. sie ließen Siedlung Siedlung sein und wählten den Weg zu den Indianern, mit denen sie fortan lebten. Solche >Evasionisten ‘ fanden bei den Stämmen offenbar freundliche, zumindest aber bereitwillige Aufnahme und häufig integrierten sie sich vollkommen in die indianischen Gemeinschaften. Die Vermutung ist nicht abwegig, daß die umfangreichen $>$ Hilfslieferungen $<$ an Brot, Korn, Fisch und Fleisch, die die unflexibleren Siedler schließlich von indianischer Seite erhielten, auch Initiativen weißer Überläufer zu danken sind und dem Eindruck, den diese Menschen auf die Indianer machten. Gelegentlich kehrte der eine oder andere >Aussteiger < zu seinen Landsleuten zurück und vemittelte Wissenswertes über indianische Sitten und Gebräuche (vgl. Billings 1976: 26).

Die Schwierigkeiten der ersten Europäer in Nordamerika, die der Bericht von George Percy exemplarisch benennt, fanden bereits im 17. Jahrhundert eine bis heute gebräuchliche >naturalisierte< Erklärung: die Kolonisten hätten sich in der Neuen Welt relativ schutz- und hilflos einer feindlichen, unbarmherzigen Wildnis ausgesetzt gesehen, die zu zähmen, zu beherrschen und rational umzugestalten in einem langwierigen, mühsamen und schmerzhaften, von zahllosen Fehl- und Rückschlägen durch-setzten Prozeß erst gelernt sein wollte. Doch wird ebenfalls im 17. Jahrhundert schon eine konträre Position formuliert: Die Versorgungsmisere ließe sich keinesfalls auf >Mangel und Unvollkommenheit des Landes` zurückführen (vgl. Billings 1976: 28), 
sondern liege im »government « begründet. Selbst im Paradies, so ein Beobachter im Jahre 1610, wäre es den Kolonisten unter der gegebenen Führung schlecht ergangen (vgl. Billings 1976: 28). Den für die transatlantischen englischen Kolonien offiziell vorgesehenen Modus der Organisation von Macht und Herrschaft, von Produktion und Reproduktion, verdeutlichen die Instruktionen der »London Company « an die ersten Siedler (vgl. Billings 1976: 19-22). Die Suche nach, der Anbau, die Herstellung von handelbaren Gütern, die in Europa gutes Geld bringen oder zu bringen versprechen, werden als primäre Ziele des Unternehmens definiert, denen alle anderen Aktivitäten und Operationen ausnahmslos unterzuordnen sind. Die Sorge für die Profite der Handelsgesellschaft muß absoluten Vorrang haben. Ihr soll sich der Großteil der Siedler unverzüglich und eifrig widmen. Lediglich einer kleinen Gruppe wird aufgetragen, Boden zur Deckung des Eigenbedarfs der Kolonie zu kultivieren. Fehlende Nahrungsmittel hat man von den Eingeborenen im Tausch oder sonstwie zu besorgen. Es gilt, die Siedler zusammenzuhalten und zu disziplinierter und fleißiger Arbeit für den Export anzuleiten. Zum Zwecke optimaler Kontrolle sollen Siedlungen so angelegt werden, daß von einem zentralen Punkt (Marktplatz) aus alle Straßen gut einzusehen sind.

Skizzierte Anweisungen stehen exemplarisch für eine Fülle von Rezepturen, Projekten und Programmen, die Großhandelskaufleute im Verein mit Teilen des Adels und gestiitzt auf die Staatsmacht in den europäischen Hauptquartieren mit Blick auf den Kolonisationsraum Amerika entwerfen und gehören in den Kontext von »[...] Überlegungen und Praktiken, die sich auf das gesamte 17. Jahrhundert [...] verteilen und die man unter dem ziemlich approximativen Begriff >Merkantilismus< zusammenfaßt« (Foucault 1978: 220).

Die Entdeckungs- und Eroberungszïge der Spanier im mittel- und südamerikanischen Raum galten noch primär der Suche nach Gold und Silber. Die spanische Kolonialpolitik beschränkte sich weitgehend auf die Lösung des Problems, Ausbeutung, Vermünzung und Verschiffung der mexikanischen und peruanischen Edelmetallvorkommen möglichst effizient zu organisieren. Im ökonomischen Denken des 16. Jahrhunderts war das >schöne Metalk in sich Merkmal und Ausdruck des Reichtums.

»Sein ihm eigener Glanz zeigte zur Genüge an, daß es gleichzeitig verborgene Präsenz und sichtbare Signatur aller Reichtïmer der Welt war. Aus diesem Grund hatte es seinen Preis. Aus diesem Grund maß es auch alle Preise. Aus diesem Grund konnte man es auch gegen alles, was einen Preis hatte, austauschen. Es war das Kostbare par excellence« (Foucault 1978: 220).

Im Laufe des 17. Jahrhunderts dagegen gewinnen Spekulation und Reflexion über die Natur der Reichtümer eine andere Qualität. Zwar werden im Rahmen der sich entwickelnden merkantilen Formation dem Gold bzw. dem Silber die genannten drei Eigenschaften auch weiterhin zugewiesen, man »[...] läßt sie aber nicht mehr auf der ersten Eigenheit (einen Preis zu haben) sondern auf der letzten (an die Stelle dessen zu treten, was einen Preis hat) beruhen« (Foucault 1978: 220). Das heißt die Tauschfunktion wird Grundlage für die beiden Fähigkeiten, zu messen und einen Preis zu haben. Die Charakteristika von Geld und Reichtum erscheinen nicht länger durch den immanenten Wert des (gemünzten) Edelmetalls determiniert, sondern durch Handel 
und Austausch. Der Merkantilismus läßt sich als überlegte Gliederung begreifen »[...] die aus dem Geld das Instrument der Repräsentation und der Analyse der Reichtümer und umgekehrt aus dem Reichtümern den vom Geld repräsentierten Inhalt macht « (Foucault 1978: 221). Die Korrespondenzen zwischen Geld und Reichtum liegen in der Sphäre des Zirkulationsprozesses und ergeben sich nicht weiter aufgrund der Kostbarkeit des Metalls.

»Wenn die Güter zirkulieren können (und zwar dank dem Gelde), vervielfachen sie sich und der Reichtum nimml zu« (Foucault 1978: 226).

Das ökonomische Denken des 17. Jahrhunderts setzt den Warentausch, den Handel als ausschlaggebendes, Werte und Reichtümer schaffendes Mornent (vgl. auch Malynes 1622: A 3 f.). Damit Handel möglich wird, muß an einem Ort ein Überfluß vorhanden sein, der an einem anderen Ort Bedürfnisse befriedigen kann (vgl. Malynes 1622: 95).

»Die Frucht, nach der mich hungert, die ich pflücke und die ich esse, ist ein Gut, das mir die Natur bietet. Es wird keinen Reichtum geben, wemn die Früchte auf meinem Baum nicht zahlreich genug sind, um meinen Appetit zu übersteigen. Dabei muß außer mir noch jemand Hunger haben und nach ihnen verlangen." (Foncault 1978: 242)

Nun bringt die Natur Güter von handelbarem Zuschnitt und in handelbarem Umfang nur bedingt spontan hervor. Ihr muß entsprechend nachgeholfen werden. Die >Merkantilisten< konstatieren die Notwendigkeit, zur Förderung der Naturproduktion Geld und Arbeit in den Boden zu investieren. (Dabei ist Arbeit nicht als wertschöpfend und Geld nicht als allgemeines Äquivalent geronnener Arbeit erkannt. Doch obwohl im >Merkantilismus die Arbeit als ökonomische Kategorie faktisch nicht existiert, impliziert die Einschätzung des Tausches als Reichtumsspender eine Steigerung ihrer Wertschätzung; vgl. Marx 1977: 349 u. 793; Fusfeld 1975: 29). Es sind politische Garantien gefordert, die ein ausreichendes Angebot von Arbeitskräften zu sichern vermögen und die gewälhrleisten, daß Vorschüsse und Rückzahlungen im Verhältnis zum Boden kalkulierbar stattfinden können und daß die Einsätze lohnen.

Damit sich die Investitionen der Kaufleute amortisieren und die anvisierten Zirkulationsgewinne realisieren, bedarf es abgesicherter und verläßlicher Handlungsketten über zum Teil erhebliche zeitliche und räumliche Distanzen. Die erfolgversprechende Planung, Installierung und Aufrechterhaltung solcher Handlungsketten ist abhängig von der Herausbildung zentralisierter Autoritäten, von der Schaffung einer stabilen Herrschaftsorganisation, d. h. hierarchisierter Koordinations- und Regulationsorgane, und auf subjektiver Ebene von einer >Anpassung des inneren Überbaus < an die den neuen ökonomischen Tendenzen entsprechenden gesamtgesellschaftlichen Erfordernissen.

»Das Verhalten von immer mehr Menschen muß aufeinander abgestimmt, das Gewebe der Aktionen immer genauer und straffer durchorganisiert sein, damit die einzelne Handlung darin ihre gesellschaftliche Funktion erfüllt. Der einzelne wird gezwungen, sein Verhalten immer differenzierter, immer gleichmäßiger und stabiler zu regulieren« (Elias 1979: 317).

Die Bändigung der >äußeren Natur< findet ihre Entsprechung in der Bändigung der sinneren Natur<. Die imperial-merkantile Okkupation und Nutzbarmachung der 
Territorien läuft parallel zu einer imperial-merkantilen Okkupation und Nutzbarmaclung der menschlichen Körper und Gehime.

Die Anforderungen, die reibungsloser Fluß und Kontinuität des Handels stellen, fiihren allmällich zur Ausprägung relativ beständiger zentraler Monopolinstitute der Macht- und Gewaltausübung und zı einer Veränderung des Standards des gesellschaftlich Geforderten und Verbotenen. Der wesentlich durch substistenzwirtschaftliche Orientierungen geprägte prämerkantile Standard, der unter anderem den Zwang einer allzu langen Berechnung für die Zukunft und der Regelung der individuellen Affekte über das $\mathrm{Maß}$ der zur eigenen unmittelbaren Reproduktion erforderlichen Arbeit hinaus kaum kennt, ist für die Ambitionen und Intentionen der merkantilen Ökonomie hinderlich und wird bekämpft.

Vor allem die kaufmännischen Funktionen in den Zentren der expandierenden Fernhandelsverflechtungen gewöhnen »[...] an eine Unterordnung der augenblicklichen Neigung unter die Notwendigkeit der weitreichenden Interdependenz « (Elias 1979: 338). Im Zuge der weiteren Entwicklung geht eine Ausweitung des >effektiven Lebensraumes $<$ mit einer zunehmenden Reduktion des >beherrschten Lebensraumes< konform. Subjektive Kompetenzen und objektive Möglichkeiten einer autonomen Selbstversorgung werden freiwillig oder unter $Z$ wang aufgegeben und geraten in Vergessenheit. Immer mehr soziale Akteure sehen sich in ihrer Daseinsfürsorge abhängig von zentralisierten Instituten und hierarchisch organisierten Netzen der Logistik, der Versorgung, der Verteilung. Immer mehr soziale Akteure auch erscheinen vom Verkauf und nicht mehr länger vom direkten Verbrauch ihrer Erzeugnisse abhängig. Das Handelsbürgertum forciert von Anfang an und mit zunehmend repressiven Mitteln eine Ausdehnung merkantilistischer Normen und Maximen der Produktion und der Reproduktion auf alle Schichten und Räume.

Zur vollen Entfaltung gelangt der Merkantilismus mit der Errichtung und Ausbeutung eines abhängigen Kolonialsystems. Entsprechend dem Postulat merkantiler Handelstheorien, daß der Wert der Exporte eines Landes den seiner Importe übersteigen muß, damit sich nationaler Reichtum akkumulieren kann (vgl. Bruchey 1966: 46), erhalten die Kolonien eine doppelte Funktion zugewiesen. Sie sollen das Mutterland mit agrarischen und sonstigen Rohstoffen beliefern und ihm fertige (Manufaktur-)Erzeugnisse abnehmen (vgl. auch Malynes 1622: 95). Dabei versuchen die europäischen Mächte, in den von ihnen beanspruchten und besetzten auswärtigen Territorien speziell autorisierte Kaufleute und Handelsgesellschaften als Monopolkäufer und -verkäufer zu etablieren und die Austauschbedingungen exklusiv zu bestimmen. Das geht z.B. so weit, daß in den Kolonien die Ausübung bestimmter Berufe und Einrichtungen bestimmter Produktionsstätten und Produktionszweige verboten wird, um den Ausfuhrgütern des Mutterlandes keine Konkurrenz erwachsen zu lassen. Die kolonialen Außenhandelsmärkte entwickeln sich so mit der Zeit zu einer Art sinstitutionalisierter Produkte «: »sie resultieren aus politischen Anstrengungen und militärischer Gewalt« (Habermas 1979: 31; vgl. auch Dobb 1970: 210).

Die gängigen historiographischen Abhandlungen zum Prozeß der `Eroberung « Nordamerikas durch die Europäer pflegen bis auf den Tag Investoren und Siedler, Ko- 
lonisatoren und Kolonisten quasi auf eine Stufe zu stellen und wie mehr oder weniger gleichberechtigte, vor allem aber gleichgesinnte Partner zu behandeln. Bei (unterstellter) weitgehender Übereinstimmung der Motive und Intentionen beider Gruppen, nämlich möglichst schnell möglichst hohen materiellen Gewinn zu erzielen, investiert die eine ihr Geld, die andere entsprechend ihre Arbeitskraft. Dabei wird geflissentlich übersehen oder verschwiegen, daß zu Beginn des 17. Jahrhunderts die merkantil geprägte Welt- und Wirklichkeitsauffassung samt ihrer Definitionen vom Sinn und Nutzen wirtschaftlicher Tätigkeit den Status einer weitgehend allgemeinen Gültigkeit und Verbindlichkeit noch längst nicht erreicht hat. Viele, wenn nicht gar der überwiegende Teil der Menschen, die gerade in der Anfangsphase europäischer kolonialer Unternehmungen auf dem nordamerikanischen Festland freiwillig oder gezwungen ihren Weg in die Neue Welt nehmen, gehören jenen Klassen und Schichten der europäischen Gesellschaft an, die der beginnende Zersetzungsprozeß des Feudalismus aus der Bahn ihrer traditionellen Lebensweise wirft, ohne ihnen unmittelbar neue Chancen zu eröffnen. Wir haben es mit Gruppen zu tun, die sich zwar der »[...] Notwendigkeit eines qualvollen Anpassungsprozesses an die Bedingungen des Geldkapitalismus [...] (Borkenau 1932: 315) gegeniibersehen, dieser Notwendigkeit aber nicht entsprechen können oder nicht entsprechen wollen. Das Denken und Handeln dieser Pioniere, ihr Wahrnehmungs- und Sinngebungsmodus, bleiben weiterhin wesentlich von eher substistenzwirtschaftlichen Orientierungen geprägt und sind nicht vom Streben nach Surplus und Profit beherrscht. Zumindst aber sind etlichen Kolonisten solche Orientierungen noch nicht so fremd, als daß sie im Kontakt mit den sarchaischen $<$ Vorstellungen und Praktiken der Indianer nicht schnell wieder auflebten. Auch auf der Ebene der Selbstdisziplinierung entspricht das Verhalten des >kolonialen Fußvolkes< keinesfalls den Standards der merkantilen Elite.

Die (Weiter-)Existenz und die Virulenz subsistenzwirtschaftlicher Orientierungen mit all ihren Implikationen und Konsequenzen (vgl. Groh 1987; Bardelle 1988) macht den Kolonisatoren (auch) jenseits des Atlantiks erheblich zu schaffen. Dies verdeutlicht z.B. die Serie strenger Vorschriften und Gesetze, die auf Betreiben der »London Company « im Jahre 1611 für Virginia erlassen und unter der Bezeichnung »Laws Divine, Morall and Martiall« bekannt wird (vgl. Billings 1976: 29-33). Kein Kolonist, so die eindeutige Order, darf die Siedlung ohne schriftliche Genehmigung verlassen. Nicht ausdrücklich von der Kolonialadministration autorisierte Beziehungen zur indianischen Bevölkerung sind unter Androhung der Todesstrafe verboten. Über Art und Umfang selbstbesorgter Nahrungsmittel wird genaueste Rechenschaft gefordert. Ähnliche Statuten ergehen auch in den anderen europäischen Kolonien in Amerika. All diese Regulierungsmaßnahmen laufen darauf hinaus, die Fülle und Vielfalt der nach den Gegebenheiten des Raumes möglichen autonomen menschlichen Lebensäußerungen auf ein überschaubares und ausbeutbares Spektrum zu reduzieren. Das schließt den zerstörerischen Zugriff auf den Raum selber ein. Was wild wächst und wuchert - und zumindest tendenziell freie Reprodukion zu garantieren vermag - wird umgehauen oder untergepflügt. Die natürlichen, artenreichen Mischkulturen müssen künstlichen, anfälligen und auslaugenden Monokulturen weichen. All diese Regulie- 
rungsmaßnahmen sind Teil der imperialen Reaktion auf zahllose Fälle von Insubordination, Gehorsamsverweigerung, Desertion und Meuterei, die immer wieder den Bestand fester Siedlungen, die Durchsetzung der merkantilen Ordnungs- und Ausbeutungskonzeption samt ihres spezifischen, tauschwertorientierten Arbeits- und Handelsethos, die Produktion und Aneignung von (exportierbaren) Überschüssen, die Realisierung kommerzielle Profite gefährden.

In diesem Zusammenhang ist die Vermutung keinesfalls abwegig, daß die Forts, die überall aus dem Boden schießen, nicht primär die Funktion haben, >böse Wilde< abzuwehren, sondern unzufriedene Zivilisierte am Weggehen zu hindern.

\section{Aussteiger und Selbstversorger}

Die traditionelle Perspektive der amerikaspezifischen Kolonial geschichtsschreibung grenzt bis heute weitgehend jene Europäer aus, die in die Neue Welt nicht die Gier nach Gold und schnellem Reichtum trieb, sondern eher die greifbare Chance, Träume von einer freien, selbstbestimmten und selbstgenügsamen Existenz zu >erden< und zu leben. Statt ihre Kraft und Energie darauf zu konzentrieren, die vorgefundenen natürlichen Ressourcen möglichst schnell und effektiv in private Gewinne zu verwandeln, tauchten zahlreiche Pioniere ein in die Ökologie des Raumes und die einer >indianischen $<$ Lebensweise und gingen zum Teil gänzlich in ihnen auf. Schon eine der ersten englischen Siedlungen, die Raleigh auf der Insel Roanoke in den >Carolina Outer Banks< gründete, wurde im Jahre 1590 verlassen vorgefunden, ohne daß irgendein Anzeichen für einen gewaltsam erzwungenen Aufbruch der Kolonisten sprach. In einen Holzpfahl eingekerbt entdeckte man das Wort >Croatoan<, das wohl die letzte Gruppe bei ihrem Verschwinden zurückließ; ein Hinweis auf das mögliche Ziel des damals wie heute geheimnisumwitterten Exodus. Crotoan ist der Name einer anderen Insel der besagten Banks. Kein einziger der >verlorenen< Siedler tauchte jemals wieder auf. $\mathrm{Zu}$ vermuten steht, daß sie mit den Indianern zogen, da ihnen dies bessere Lebensperspektiven bot als das Warten auf Nachschub aus dem Mutterland und die Aussicht, für die Profite von Kaufleuten zu arbeiten.

Trotz ständig ausgreifender merkantiler Okkupation und Territorialisierung kann Nordamerika bis weit ins 17. Jahrlhundert hinein als Raum gelten, in dem die Verhältnisse zu einem hohen Grad dezentral und unbestimmt sind. Die europäischen Pioniere sehen sich einer auswegreichen Situation gegenüber, die es ihnen aufgrund einer Fülle offener Möglichkeiten für eine unabhängige und selbstbestimmte Reproduktion lange Zeit erlaubt, einzeln oder in Gruppen die Subordination unter einen zentralisierenden, disziplinierenden und vereinheitlichenden Apparat und (im merkantilen Sinn) rational geplante Arbeits- und Ausbeutungsverhältnisse erfolgreich zu verweigern, ihre Autonomie zu wahren und für staatliche und kommerzielle Zwecke unverfügbar zu bleiben.

Unausrottbar erscheint in der Literatur zum Thema der Topos, die Begegnung der Europäer mit Amerika im Anschluß an die erste >Indienfahrt « des Columbus sei die Begegnung mit einer bis dahin noch nicht erfahrenen, fremden Welt gewesen; (als 
Beispiel aus jüngster Zeit s. Gewecke 1986: 56). Bei näherem Hinsehen offenbart sich, daß Flora und Fauna Nordamerikas ebenso wie der Existenzmodus und die Denk- und Wahrnehmungsweise der Indianer wohl graduell, keinesfalls aber prinzipiell von dem unterschieden sind, was vielen der ersten Kolonisten seinerzeit aus der europäischen Tradition und Gegenwart vertraut ist. Damit soll nicht bestritten werden, daß die Neue Welt vor allem in klimatischer Hinsicht auch einige Überraschungen für die Töchter und Söhne der Alten Welt bereithielt.

Zur Zeit der ersten Kontakte mit Weißen praktiziert die einheimische nordamerikanische Bevölkerung ein breites Spektrum vielfältiger Methoden der Subsistenzsicherung entsprechend der Dargebote und Konditionen der jeweiligen lokalen Ökosysteme. So betreiben die sogenannten Pueblo-Indianer intensiven Anbau in einer ariden Umgebung, die halbnomadischen Indianer der Plains ernähren sich hauptsächlich von wilden und teilweise von kultivierten Kornpflanzen, im Great Basin und in den Plateau Regions bilden Nüsse, Beeren und Wurzeln, an der Nordwest-Küste Flußund Seefische und Vögel die Basis der Ernährung. Im ausgedehnen Waldland des Ostens, dort, wo die Europäer zunächst Fuß fassen, leben die Indianer als Farmer, Jäger, Fischer und Sammler. Insgesamt ist die Ernährung weitgehend vegetarisch orientiert. Lediglich aufgrund eines männlichen Bias, der sich bis heute in viele Forschungen und Betrachtungen zur indianischen Lebensweise einschleicht, erhalten die Jagdaktivitäten eine herausragende Bedeutung zugewiesen (vgl. Vecsey/Venables 1980: ix-xxv). Die Subsistenztechnologie der Indianer kennt keine Zugtiere, keine Pflüge und keine Räder. Doch verstehen sie es sehr wohl, durch eine Fülle von Eingriffen und Manipulationen die natürlichen Erträge der Nähr- und Nutzpflanzen zu wahren und auch zu vermehren. Alles in allem werden über 150 Pflanzensorten für Nahrung, Kleidung und Heilung gehegt und kultiviert (vgl. Vecsey 1980; Jennings 1974: 129-132; Castetter 1935; Anderson 1952). Es gibt begründete Vermutungen, daß im 17. Jahrhundert die Hektarerträge indianisch >bewirtschafteten< Landes teilweise höher lagen als entsprechende Erträge in Europa zur gleichen Zeit. Auch ist angenommen worden, daß die Bevölkerungszahl Nordamerikas vor der >Entdeckung der Westeuropas durchaus gleichkam (vgl. u. a. Jacobs 1980: 47). Unabhängig davon, wie man zu solchen Thesen steht, die weder eindeutig beweisbar noch eindeutig zu widerlegen sind (und das auch bleiben werden), gilt es anzuerkennen, daß die Indianer über Jahrtausende aufmerksamer Beobachtung und kluger Experimente Methoden und Techniken entwickelt und entfaltet haben, die Millionen von Menschen keinesfalls karg oder knapp am Leben erhielten, ohne das Land und seine Ressourcen nachhaltig zu schädigen.

Auch die Kenntnisse, Fähigkeiten und Fertigkeiten vieler der ersten Kolonisten sind eher auf Garten- denn auf Ackerwirtschaft orientiert. Ihnen geht es vor allem darum, sich selbst in eigener Verantwortung zu versorgen. An der systematischen, energieintensiven und tendenziell zerstörerischen Produktion handelbarer, gewinnträchtiger agrarischer und sonstiger Güter und Überschüsse haben sie wenig Interesse. Ihre Auffassung vom Charakter bäuerlicher Arbeit ist noch nicht in einem Maß um Pflug und Axt konzentriert, als daß sie die Logik und Rationalität der indianischen Lebens- 
und Wirtschaftsweise nicht schnell begriffen und für sich nutzbar machten. So sehen z.B. die ersten Dörfer, die von Engländern in Virginia angelegt werden, nicht aus wie Dörfer in England, sondern wie Dörfer der Algonkin. Die indianischen Vorstellungen von einer beseelten Natur sind dem europäischen Denken ebenfalls nicht fremd. Reflex und Pendant finden sie in der abendländischen Sagen- und Märchenwelt: Hier sprechen und handeln Tiere wie Menschen, Menschen können sich in Tiere verwandeln und Tiere in Menschen, Pflanzen und Mineralien erscheinen >animiert< usw. Auch auf dieser Ebene bewegen sich die europäischen Pioniere - die einen mehr, die anderen weniger - in durchaus bekannten Gefilden. Die tradierten Berichte aus der Frühzeit der Kolonisation erlauben den Schluß, daß die auf die vorgefundene Ökologie des nordamerikanischen Raumes bezogene Adaption und Integration denjenigen europäischen Menschen am ehesten gelingt, die im >Prozeß der Zivilisation< noch nicht zur Spitze des merkantilen Niveaus $>$ fortgeschritten $<$ sind.

Gelungene Synthesen zwischen indianischer und europäischer Kultur und Tradition erzeugen ein Milieu, in dem eigensinniger, anarchischer Widerstand gegen die imperialen Zugriffe der merkantilen Kolonisatoren wurzelt und gedeihen kann. Wer die Wälder, Berge und Prärien kennt wie die Indianer, wer sich als Eingeweihter in ihnen zu bewegen und zu unterhalten versteht, ohne allzu gravierend als Störfaktor zu wirken, ist für alles, was Stadt und Staat repräsentieren, für jede hierarchische Organisation, jede zentralisierte Ausbeutungsordnung kaum zu fassen und zu gewinnen und wird in letzter Instanz unkalkulierbar bleiben.

Ein gutes Beispiel für eine Existenzweise an der Peripherie bzw. jenseits der durch merkantilen Ackerbau und merkantilen Handel bestimmten Grenzen liefern die Waldläufer und Trapper Kanadas. Wiewohl vieles für die Behauptung spricht, daß diese Menschen $\gg[\ldots]$ aus den unzugänglichen Wäldern die Werte erst herbeigeschafft haben, welche dazu dienten, der Besiedlung [...] die Wege zu ebnen « (Hasenclever 1923: 114) und sich letztendlich als Schrittmacher der (merkantilen) Kultur wenn auch ohne es. zu wollen - selber nach und nach den Ast absägten, auf dem sie saßen, folgt ihr Tun Mustern, die den Maßgaben merkantiler Rationalität und Zweckbestimmung weitgehend nicht entsprechen. Das Verhalten der Waldläufer und Trapper läßt deutlich Spuren einer Ökonomie erkennen, die der >Verzehrung der Reichtümer< Vorrang einräumt vor ihrem Erwerb und ihrer Anhäufung/Vermehrung (vgl. Bataille 1975: 35). Sie verschwinden auf ein Jahr oder länger in den Wäldern, um dann ihre Beute an Fellen, gelegentlich auch an getrocknetem Fleisch, auf den Märkten der festen Siedlungen anzubieten. Aus den Erträgen ihrer Expeditionen bestreiten die Trapper/Waldläufer den Minimalverbrauch zur Erhaltung des Lebens und zur Fortsetzung ihrer Profession. D. h. sie ergänzen verschlissene oder verbrauchte Gegenstände wie Pulver, Blei, Messer, Gewehre etc. Etwaige Überschüsse werden nicht gespart und nicht investiert, nicht zur Ausweitung der Ausrüstung und Unternehmungen verwandt, sondern in exzessiven Verschwendungsorgien systematisch unproduktiv verausgabt. Die so bestimmte spezififsche Pragmatik des Umganges mit Ressourcen und Surplus läuft in unbehelligter Form darauf hinaus, daß eine Akkumulation von Reichtümern, ein >Wachstum der Produktivkräfte<, eine Expansion der 
Operationen nicht stattfindet und daß der ökologische Status des Raumes, der den Waldläufern/Trappern ein relativ unkompliziertes Leben und Überleben ermöglicht, in nicht so extremer Form angegriffen wird, als daß er sich nicht regenerieren könnte. Allerdings ist zu bedenken, daß sowohl die sproduktive< Tätigkeit als auch die Verausgabungspraktiken der Waldläufer und Trapper mit der Zeit nur noch bedingt autonom sind und von der ausgreifenden >merkantilen Maschine « mehr und mehr okkupiert, manipuliert und korrumpiert erscheinen. In Bars und Bordellen werden die verschwenderischen Ausgaben kanalisiert und für die merkantile Wirtschaft nutzbar gemacht. Auch trägt die nicht unwesentlich von Waldläufern und Trappern vermittelte und forcierte Integration des >Roten Mannes < in den Pelzhandel der Weißen massiv zur Zerstörung indianischen Lebensraumes und indianischer Lebensart bei. Nichtsdestotrotz aber werden die Waldläufer und Trapper gerade auch in ihrem engen Kontakt zu den Indianern als permanente Provokation und Bedrohung begriffen und bereits im 17. Jahrhundert entsprechend diskreditiert. Ihr ungeordnetes, jeder Zucht bares Auftreten findet sich angeprangert, ihre Sitten- und Ziigellosigkeit, ihr fehlender Sinn für nationale Loyalität, ihre Ignoranz gegenüber König und Vaterland (vgl. Hasenclever 1923; Bitterli 1976: 39-42). Ihr Beispiel gilt als >gefährliche< Verlokkung für die zum Teil gepreßten und übel gebeutelten seßhaften Bauern und Handwerker in den festen Siedlungen. Waldläufer und Trapper, sonstige unabhängige Pioniere und die einheimische nordamerikanische Bevölkerung stellen als Repräsentanten, später als Relikte einer anderen, nicht-merkantilen Wirklichkeit die Guiltigkeit der Maximen bürgerlich-merkantiler Sittlichkeit und Rationalität grundsätzlich in Frage. Die Synthesen zwischen indianischer und europäischer Kultur bergen ein >Stück Utopie <: die autonome Reproduktion auf der Basis nicht-paternalistischer, nicht-zentralisierter Organisatonsstrukturen sozialen Seins außerhalb der von Merkantilisten favorisierten, propagierten und erzwungenen Modi der in eine hierarchische Ausbeutungsordnung eingebetteten, seßhaften agrikolen und manufakturellen Arbeit und des tauschwertorientierten Handels. Darüber hinaus verweisen solche Synthesen auf die Möglichkeit eines alternativen Umgangs mit den nordamerikanischen Ressourcen, der sich wesentlich auf die Befriedigung energetischer Bedürfnisse, d. h. auf die Wiederherstellung verausgabter Energien beschränkt, den ökologischen Systemen Zeit und Raum zur >natürlichen< Regeneration läßt und den Sinn von Reichtum und Überfluß in seiner unproduktiven Verausgabung sieht. Nicht zuletzt Waldläufer und Trapper halten die Erinnerung an diese andere Möglichkeit der Wirklichkeit wach, während die merkantile Ordnung zwecks Optimierung ihrer Funktionsfähigkeit, der Akkumulations- und Investitionsbedingungen der Reichtïmer, alle Reminiszenzen an Orientierungen subsistenz-wirtschaftlicher Art und Provenienz zu unterdrücken und vergessen zu machen trachtet.

Der am Ende erbittert geführte Kampf des im nordamerikanischen Kolonialgeschäft engagierten europäischen Handels- und Finanzbürgertums gegen $>$ Indianer $<$ roter wie weißer Hautfarbe ist Teil einer umfassenden Strategie, die über »ständige Zensur« dafür sorgen muß $»[\ldots]$ daß das Andere die schöne Ordnung der Realität, des Diskurses, der Rationalität nicht durcheinanderbringt« (Böhme 1983: 13). 


\section{Zur Aktualität der imperial-merkantilen Perspektive}

Bereits im 17. Jahrhundert verleiht die merkantilistische Propaganda den kolonialen Unternehmungen in Amerika den Charakter einer zivilisatorischen Mission. So wird ideell Grund und Recht für die merkantile Expansion geschaffen mit dem Ziel, ihren Fortschritt zu sichern. Das Muster der Argumentation sei kurz skizziert: Die Pioniere, die im späten 16. und frühen 17. Jahrhundert in die Neue Welt gelangen, kommen aus hohen Kulturen, aus den in ihrer politischen und ökonomischen Entwicklung fortgeschrittensten Ländern Europas. Allerdings bewegen sie sich zum großen Teil in diesem Rahmen auf sehr niedrigem sozialen und moralischen Niveau, gehören zum >Bodensatz< der Gesellschaften der Alten Welt. In den Wildnissen (Nord-)Amerikas zunächst weitgehend auf sich selbst gestellt und der eigenen Willkür überlassen, verrohen diese ohnehin unsicheren Kantonisten notgedrungen vollends und sinken rasch in eine Art Uızustand zurïck. Ihre Manier zu wohnen, sich zu kleiden und zu ermähren, die Formen ihres Umgangs miteinander und mit den Dingen weichen eklatant von Standards ab, die Normalität und Zivilisation konstituieren und lassen deutlich Elemente erkennen, die primitiven, in der Alten Welt eigentlich bereits überwundenen Entwicklungsstufen soziokultureller Evolution entsprechen. So zerfallen die ersten Europäer, die sich in Amerika dauerhaft aufhalten, in kleine, voneinander isolierte, plan- und ziellos umherschweifende Gruppen. Sie leben in einem anarchischen $\mathrm{Zu}-$ stand ohme Ordnung, ohne Führung, ohne Ehrgeiz (außer dem, irgendwie zu überleben), ohne nennenswerten Privatbesitz, ohne Spartätigkeit mehr schlecht als recht von der Hand in den Mund. Sie betreiben Jagd und Fischfang, kaum Ackerbau, sammeln wilde Früchte und haben bei sporadisch auftretendem Überfluß nichts als den augenblicklichen Genuß im Sinn. Sie zeigen einen ausgeprägten Hang zu unproduktiven Lebensäußerungen und lassen ein positives Verhältnis zu geregelter und disziplinierter Arbeit weitgehend vermissen. Eine erfolgreiche und profitable Bewirtschaftung des Landes, eine adäquate Exploration, Entwicklung und Realisierung der ökonomischen Potentiale des (nord)-amerikanischen Raumes, ist auf solch desolater Basis natürlich ausgeschlossen. Doch Rettung naht. Als Avantgarde des Fortschritts und der Zivilisation, durch Rückschläge und harte Erfahrung gestählt, nehmen sich Kaufleute der nicht ganz leichten und ehrenvollen Aufgabe an, ihre abgedrifteten Landsleute in den transatlantischen Territorien in die Bahnen europäischer Gesittung zurückzugeleiten. Durch eine hauptsächlich von führenden Vertretern des Handelsund Finanzbürgertums betriebene und verantwortete Politik gelingt es nach und nach, in (Nord)-Amerika Strukturen zu schaffen und fest zu etablieren, die alle Arten von Sicherheit und Prosperität begünstigen. So können schließlich unter den segensreichen Auswirkungen der merkantile Initiativen weite Landstriche urbar gemacht und die Bodenerträge gesteigert werden, können feste Ansiedlungen, urbane Zentren, geordnete Familien und regelmäßiger Waren-, Güter- und Geldverkehr entstehen und sich entfalten.

Soweit im großen und ganzen das Bild, das sich in der tradierten dokumentarischen Masse des 17. Jahrhunderts gezeichnet findet. Historikerinnen und Historiker der fol- 
genden Jahrhunderte haben bis in die Gegenwart die Essentials der imperial-merkantilen, eurozentrischen Selbstdarstellung - von wenigen Ausnahmen abgesehen weitgehend unhinterfragt uibernommen. Amerika erscheint auch heute noch als Kontinent, der gründlich aus- und aufgeräumt werden mußte, um in einen für europäischen Menschen trag- und nutzbaren Zustand zu gelangen.

Als ein Beispiel unter vielen mögen folgende Zitate für sich selber sprechen:

»Die ersten Siedler in dem Gebiet, das heute die Vereinigten Staaten bedeutet, fanden sich auf der ganzen langen Strecke von Maine bis Florida einem Wald gegenüber [...] . Verstreut über diesen Wald waren ein paar hunderttausend Eingeborene, Indianer. Sie waren beschränkt in ihren Anpassungsmöglichkeiten durch die [...] Armut ihrer natürlichen Umgebung und durch ihre Unfähigkeit, diese Umgebung zu verändern. Sie hatten keine Pferde, kein Vieh; sie mußten Jäger und Pflanzer sein, und es war unmöglich, auf dieser Grundlage eine an Zahl große und wachsende Gesellschaft in den Wäldern zu schaffen. [...] Bevor eine amerikanische Gesellschaft von den Siedlern geschaffen werden konnte, mußte Amerika umgeformt werden. Der Wald mußte Feldern weichen; der Pflug, der Zugochse, das Maultier, das Schaf, die Ziege, das Rad, das Schießpulver, der Stahl mußten ein neues Leben möglich machen, in welchem viele Millionen im Überfluß leben konnten, wo wenige hunderttausend Wilde ein ungesichertes und armes Leben hatten« (Brogan 1947: 13).

»[...] die frühen Siedler hatten es lange nötig, sich Fähigkeiten zu erwerben, die den Fähigkeiten der Wilden gleiclkamen, und eine Wildheit, die nicht viel geringer war« (Brogan 1947: 18).

"Manche von den ersten Ansiedlern waren halbe Nomaden [...] , inehr erpicht auf Jagd und Fischfang als auf die stetige Mühsal des Bauern [...]« (Brogan 1947: 21).

Auch heute noch wird in bezug auf Amerika die Geschichte der befestigten Plätze, der umgrenzten Räume und ihrer Expansion ins >Niemandsland erzählt und gewürdigt und die Spur der Zerstörung verschwiegen oder beschönigt, die diese Expansion hinterläßt. Wenn ein befestigter Platz der >Wildheit< weicht, wenn ein begrenzter Raum wieder entgrenzt wird, gilt das Projekt als gescheitert. Nicht gepanzerte und nicht umgrenzte soziale Akteure kommen in der Historiographie (auch in diesem Fall) nicht oder nur als Marginalien und abschreckende Kontrapunkte vor. Doch:

»Nicht in der Geschichtswissenschaft stattzufinden, heißt nicht, nicht in der Geschichte wirksam zu sein« (Rittner 1975: 113).

\section{Literatur}

Anderson, Edgar (1952): Plants, Man and Life, Boston.

Bardelle, Frank (1988): Ansätze zu einer historisch-ökologischen Betrachtung der Frülngeschichte und der Antike, in: Prokla, Heft 72, 1988/3, 137-159.

Bataille, Georges (1975): Die Aufhebung der Ökonomie. (Das theoretische Werk, Band 1), München.

Billings, Warren M. (Hrsg.) (1976): The Old Dominion in the Seventeenth Century. A Documentary History of Virginia, 1606-1689, Williamsburg (Virginia).

Bitterli, Urs (1976): Die »Wilden und die »Zivilisierten $\ll$, München.

Böhme, Harmut u. Böhme, Gernot (1983): Das Andere der Vernunft. Zur Entwicklung von Rationalitätsstrukturen am Beispiel Kants, Frankfurt am Main.

Borkenau, Franz (1932): Zur Soziologie des mechanistischen Weltbildes, in: Zeitschrift für Sozialforschung, 1. Jg. 1932, Heft 3, 311-335.

Brogan, Denis W. (1947): Der amerikanische Charakter, Stuttgart.

Bruchey, Stuart (Hrsg.) (1966): The Colonial Merchant. Sources and Readings, New York, Chicago, Burlingame.

Castetter, Edward F. (1935): Uncultivated Native Plants Used as Sources of Food, Albuquerque. 
Dobb, Maurice (1970): Entwicklung des Kapitalismus. Vom Spätfeudalismus bis zur Gegenwart, Köln, Berlin.

Elias, Norbert (1979): Über den Prozeß der Zivilisation, Soziogenetische und psychogenetische Untersuchungen, Band 2: Wandlungen der Gesellschaft. Entwurf einer Theorie der Zivilisation, Frankfurt am Main.

Foucault, Michel (1978): Die Ordnung der Dinge. Eine Archäologie der Humanwissenschaften, Frankfurt am Main.

Fusfeld, Daniel R. (1975): Geschichte und Aktualität ökonomischer Theorien. Vom Merkantilismus bis zur Gegenwart, Frankfurt am Main, New York.

Gewecke, Frauke (1986): Wie die neue Welt in die alte kam, Stuttgart.

Groh, Dieter (1987): Strategien, Zeit und Ressourcen. Risikominimierung, Unterproduktivität und Mußepräferenzen - die zentralen Kategorien von Subsistenzökonomie, in: Prokla, Heft 67, 1987/2, 7-34.

Habermas, Jürgen (1979): Strukturwandel der Öffentlichkeit. Untersuchungen zu einer Kategorie der bürgerlichen Gesellschaft, Darmstadt.

Hasenclever, Adolf (1923): Die Waldläufer Kanadas im 17. Jahıhundert, in: Preußische Jahrbücher, Band 193, Heft 2, 1923, 113-133.

Henning, Richard (1940): Columbus und seine Tat. Eine kritische Studie über die Vorgeschichte der Fahrt von 1492 , Bremen.

Jacobs, Wilbur R. (1980): Indians as Ecologists and Other Environmental Themes in American Frontier History, in: Vesey/Venables 1980, 46-64.

Jennings, Jesse D. (1974): Prehistory of North America, New York.

Jones, Gwyn (1965): The North Atlantic Saga, London.

Kamper, Dietmar (Hrsg.) (1975): Abstraktion und Geschichte. Rekonstruktionen des Zivilisationsprozesses, München, Wien.

Malynes, Gerard (1622): Lex Mercatoria or the Ancient Law Merchant, London.

Marx, Karl (1977): Das Kapital, Band 3 (MEW 25), Berlin.

Müller, Werner (1982): Amerika. Die neue oder die alte Welt? Berlin.

O’Donaghue, Denis (1893): Brendaniana, Dublin.

Relirmann, Norbert (1989): Spanien, Europa und Lateinamerika - Zur Geschichte legendärer Kulturbeziehungen, in: PROKLA, Heft 75, 1989, S. 109-131

Rittner, Volker (1975): HorkheimerlAdorno: Die Dialektik der Aufklärung. Die unterirdische Geschichte des Abendlandes und das Verhältnis von Körper, Herrschaft und Zivilisation, in: Kamper 1975, 126-160.

Steiner, Stan (1985): Der Untergang des weißen Mannes? Indianische Weissagungen und die Wirklichkeit, Reinbek bei Hamburg.

Vecsey, Christopher (1980): American Indian Environmental Religions, in: Vecsey/Venables 1980, 1-37.

Vecsey, Christopher u.Venables, Robert W. (Hrsg.) (1980): American Indian Environments. Ecological Issues, in Native American History, Syracuse, New York. 